Metal-Catalyzed

Asymmetric

Synthesis and

Stereoselective

Reactions

\title{
Enantioselective Addition of Allyl Bromides to Ketones
}

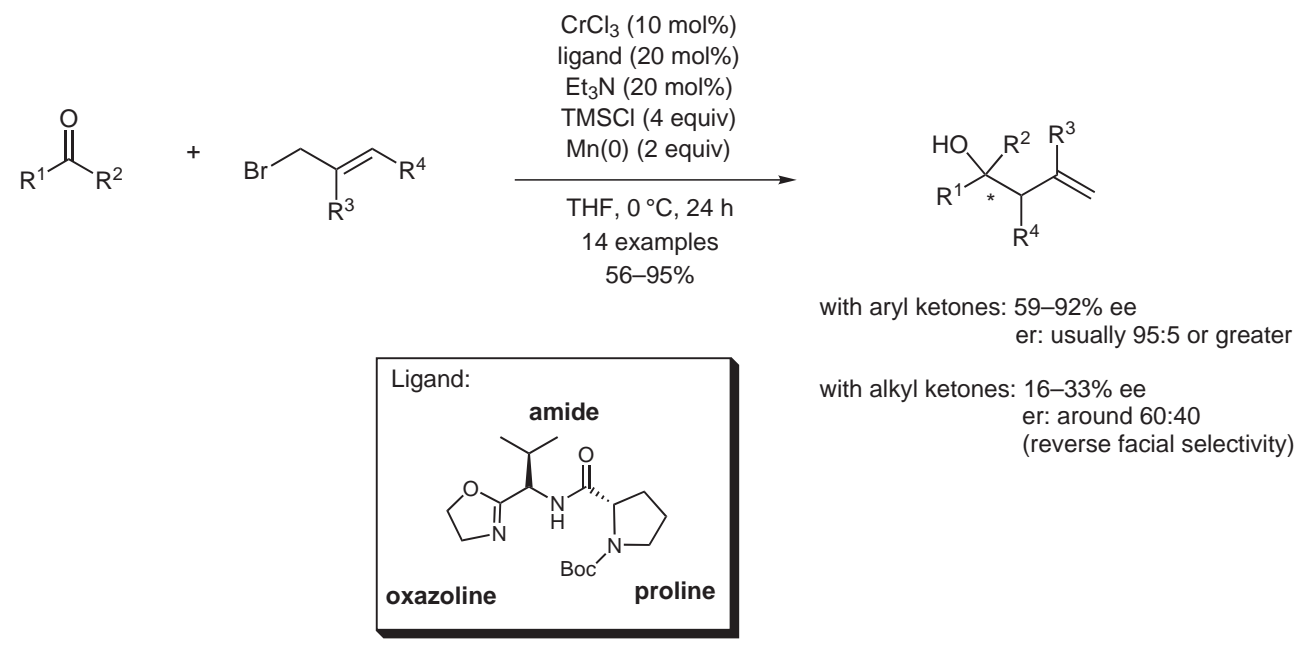

Significance: As an extension of the author's previously reported enantioselective Nozaki-HiyamaKishi reaction (J.-Y. Lee, J. J. Miller, S. S. Hamilton Org. Lett. 2005, 7, 1837-1839), this communication highlights the first example of a chromiumcatalyzed enantioselective addition of allylic bromides to ketones. The ligand used is synthesized in three steps from a protected valine. For aryl ketones, the scope is broad and encompasses various substitution types (electron-withdrawing, -donating, or neutral) and placements (ortho, meta, or para) on the aromatic ring with good to excellent ee values and yields. Halide-substituted aryl ketones are also well tolerated. Methylallyl- and crotylbromides can also be used as the nucleophilic components; modest diastereoselection (3.8:1) is seen for crotylbromide addition. Poor enantiomeric ratios are observed for aliphatic ketones and the facial selectivity is reversed.
Comment: Asymmetric addition of allyl nucleophiles to carbonyls is a popular method for constructing enantioenriched homoallylic alcohols, although this method is largely limited to aldehydes. The present report using ketones is thus a valuable contribution to this area. Moreover, using alIylic halides as the allyl source had not been reported in a catalytic version of this transformation. The success of the asymmetric process was attributed to modular catalyst design; systematic changes were made to the ligand template and the modified ligands were tested until good ee values were obtained. The authors concluded that the oxazoline module of the ligand has little effect on asymmetric induction, whereas the proline module and the relative stereochemistries of the proline and amide modules have a large effect. The relative configurations of stereocenters in the ligand affect both the enantioselectivity and the absolute configuration of the product. 